\title{
A generalization of Condorcet's Jury Theorem to weighted voting games with many small voters
}

Received: 30 June 2005 / Revised: 8 March 2007 / Published online: 4 May 2007

(C) Springer-Verlag 2007

\begin{abstract}
We extend Condorcet's Jury Theorem (Essai sur l'application de l'analyse à la probabilité des décisions rendues à la pluralité des voix. De l'imprimerie royale, 1785) to weighted voting games with voters of two kinds: a fixed (possibly empty) set of 'major' voters with fixed weights, and an ever-increasing number of 'minor' voters, whose total weight is also fixed, but where each individual's weight becomes negligible. As our main result, we obtain the limiting probability that the jury will arrive at the correct decision as a function of the competence of the few major players. As in Condorcet's result the quota $q=1 / 2$ is found to play a prominent role.
\end{abstract}

Keywords Condorcet's Jury Theorem · Weighted voting games · Majority games

JEL Classification Numbers C71 · D71

\section{Introduction}

Condorcet (1785) considers collective decision-making, where the objective is 'truth-tracking'. The fundamental premise is that there is a unique unanimously preferred alternative (the 'truth'), but voters only have partial information and imperfect competence for detecting it. The probability of a single voter's choice being correct is taken to quantify the competence of a voter. Here, the quantity of

I wish to thank Maurice Koster, Moshé Machover, Guillermo Owen and two anonymous referees for helpful comments.

I. Lindner

Utrecht School of Economics, Boothstraat 6, 3512 BW Utrecht, The Netherlands

E-mail: I.Lindner@econ.uu.nl 
interest is the jury competence of the decision-making body-the probability of arriving at the correct decision. Condorcet assumes equal individual competence, greater than 1/2, on a dichotomous choice. Condorcet's Jury Theorem shows that under simple majority rule, jury competence approaches one with increasing size of the group or increasing individual competence. Over the past decades, this celebrated result has been extended in numerous ways by statisticians, economists, political scientists, etc. ${ }^{1}$

The simple majority game as considered by Condorcet is a special case of a weighted voting game (WVG). Here, each board member is assigned a non-negative number as weight and a relative quota indicates the fraction of the total weight required for a win. The aim of this note is to provide a generalization of Condorcet's Jury Theorem to WVGs when the voters are of two kinds: a fixed (possibly empty) set of major (big) voters with fixed weights, and an ever-increasing number of minor (small) voters whose total weight is also fixed, but each individual weight becomes negligible. Using the idea that asymptotically many minor voters act like a modification of the quota for the vote among major voters, ${ }^{2}$ the limiting jury competence is derived as a function of the competence of the few major players (as a group). As in Condorcet's result, the quota $q=1 / 2$ is found to play a prominent role. We show that it maximizes the range of values of major weights for which jury competence converges to infallibility. This covers the case where major voters are absent, and Condorcet's original Jury Theorem results as a by-product.

\section{The model}

Consider a partition of the set of voters into two camps. The set of major voters is $L=\{1, \ldots, l\}$, where $l$ is a natural number. ${ }^{3}$ Each $k \in L$ is assigned a weight $w_{k}$, and let $w_{L}=\sum_{k \in L} w_{k} \in[0,1]$ denote the combined voting weight of $L$. We shall consider a sequence of WVGs $\left\{\Gamma^{n}\right\}_{n \in \mathbb{N}}$ with a growing population of minor voters. In each of these games $\Gamma^{n}$, the set of $n$ minor voters is denoted by $M^{n}=\{l+1, \ldots, l+n\}$. For each $n$, these voters have weights $\alpha_{1}^{n}, \ldots, \alpha_{n}^{n}$, which sum up to $\alpha=1-w_{L}>0$. For any coalition $S \subset L \cup M^{n}$ we interpret $w(S)$ as the aggregate voting weight of $S$.

Formally, the WVG $\Gamma^{n}$ is described by the tuple

$$
\Gamma^{n}=\left[q ; w_{1}, \ldots, w_{l}, \alpha_{1}^{n}, \ldots, \alpha_{n}^{n}\right]
$$

where $q \in(0,1]$ is the relative quota. $S$ is a winning coalition in $\Gamma^{n}$ iff $w(S) \geq q$. The latter (weak) inequality may be replaced by the strict inequality $>$. In this case we change the bracket notation in (1) to $\left\langle q ; w_{1}, \ldots, w_{l}, \alpha_{1}^{n}, \ldots, \alpha_{n}^{n}\right\rangle$.

\footnotetext{
${ }^{1}$ See e.g., Fey (2003) for references to recent work.

2 Dubey and Shapley (1979) use a similar argument for analyzing asymptotic properties of the Banzhaf index.

${ }^{3}$ Note that $l=0$ takes care of the case where $L$ is empty by the general convention that $\{1, \ldots, 0\}$ is empty.
} 
Put $\alpha_{\max }^{n}:=\max _{k \leq n} \alpha_{k}^{n}$ and $Q^{n}:=\sum_{k \leq n}\left[\alpha_{k}^{n}\right]^{2}$. Let $\left\{\Gamma^{n}\right\}_{n \in \mathbb{N}}$ evolve in such a way that

$$
\lim _{n \rightarrow \infty} \alpha_{\max }^{n} / \sqrt{Q^{n}}=0
$$

which ensures $\alpha_{\max }^{n} \rightarrow 0$ as $n \rightarrow \infty .^{4}$

\section{A generalization of Condorcet's Jury Theorem}

In a jury trial, assume a given a priori probability $\theta \in[0,1]$ that the defendant is guilty of the offense charged. This models the existence of a truth independent of the jury, yet unknown to its members. Each jury member (voter) $k$ is assumed to possess a more or less reliable perception about the truth. This degree of knowledge is modeled by $p_{k} \in(0,1)$, the judgemental competence of voter $k$. It is the probability that the voter will make the correct choice between the options 'guilty' or 'not guilty'. Assume the minor voters' choices are independent of one another and that a common $p \in(0,1)$ exists, the probability of any minor voter making the correct decision. Hence we put $p_{k}=p$ for all $k \in M^{n}$.

Jury competence is measured by the likelihood of the verdict being correct. Let $C_{I}[\Gamma]$ denote the probability of conviction, provided the defendant is guilty. Analogously, let $C_{I I}[\Gamma]$ denote the probability of acquittal in case of innocence. Jury competence then follows as

$$
C[\Gamma]=\theta C_{I}[\Gamma]+(1-\theta) C_{I I}[\Gamma] .
$$

For the moment put $\theta=1$, so that $C[\Gamma]=C_{I}[\Gamma]$ (the defendant is guilty). In the sequence of games $\left\{\Gamma^{n}\right\}_{n \in \mathbb{N}}$, we should expect that in the limit the continuous 'ocean' of randomly voting minor voters will be divided in such a way that the aggregate voting weight for conviction (the correct choice) is $p \alpha$. Consider the games

$$
\Gamma_{0}=\left[q-p \alpha ; w_{1}, \ldots, w_{l}\right] \text { and } \Gamma_{0}^{\prime}=\left\langle q-p \alpha ; w_{1}, \ldots, w_{l}\right\rangle,
$$

which are well-defined for $q \in \mathcal{J}(p):=\left(p \alpha, w_{L}+p \alpha\right)$. $\Gamma_{0}$ and $\Gamma_{0}^{\prime}$ can be considered limiting WVGs for the major players where the aggregate minor weight $p \alpha$ in favor of conviction is substracted from the quota $q$.

Let $\mathcal{B}_{l}=\left[w_{L} ; w_{1}, w_{2}, \ldots, w_{l}\right]$ denote the unanimity game among the major voters in which each voter has a veto. Let $\mathcal{B}_{l}^{*}=\left\langle 0 ; w_{1}, w_{2}, \ldots, w_{l}\right\rangle$ represent the special case where the major voters operate under what Rae (1969) has called a 'rule of individual initiative': action (conviction) can be initiated by any single individual. We will show that in the sequence of games $\left\{\Gamma^{n}\right\}_{n \in \mathbb{N}}, C_{I}$ converges to a limit depending on the quota $q$ and $w_{L}$. Figure 1 gives an illustration for $p>1 / 2$. Within the inner triangle $\mathcal{J}(p)$, the limit is the arithmetic mean of $C_{I}$ for the games defined in (4). Outside the closure of $\mathcal{J}(p)$, the influence of the major voters is 'destroyed'.

\footnotetext{
${ }^{4}$ However, it can be shown that $Q^{n}$ tends to zero so that condition (2) is stronger than $\alpha_{\max }^{n} \rightarrow 0$.
} 


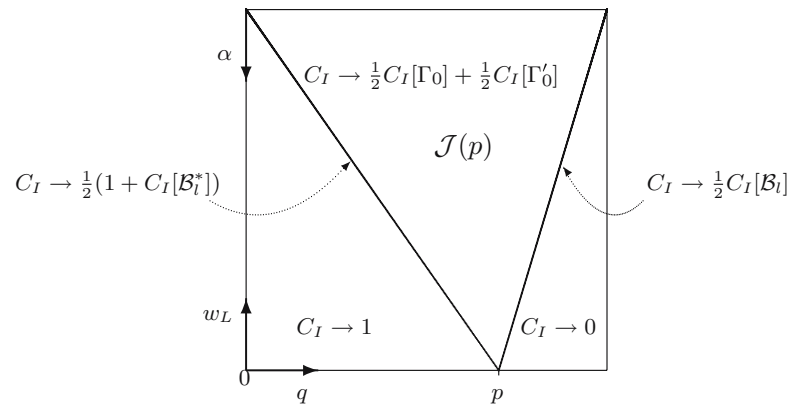

Fig. 1 Limit scenario for $C_{I}$

For $\theta=0$ (the defendant is innocent) we have $C[\Gamma]=C_{I I}[\Gamma]$. Since voting for acquittal is now correct, minor voters vote for conviction with probability $1-p$. The limit scenario of $C_{I I}$ follows analogously to $C_{I}$ by replacing $p$ by $(1-p)$ and putting $C_{I I}=1-C_{I}$. The resulting graph for $C_{I I}$ is homeomorphic to that in Fig. 1. The inner triangle $\mathcal{J}(1-p)$ is however shifted to the left.

Theorem 1 (A Generalization of Condorcet Jury Theorem to WVGs) In the sequence of WVGs $\left\{\Gamma^{n}\right\}_{n \in \mathbb{N}}$, the limiting jury competence is a function of the competence of the few major voters. In particular, jury competence converges to

$$
\lim _{n \rightarrow \infty} C\left[\Gamma^{n}\right]=\theta C_{I}+(1-\theta) C_{I I}
$$

where $C_{I}$ is given by

$$
C_{I}=\frac{1}{2} C_{I}\left[\Gamma_{0}\right]+\frac{1}{2} C_{I}\left[\Gamma_{0}^{\prime}\right], \quad \text { if } q \in \mathcal{J}(p) .
$$

For other values of $q$, the right-hand side of (6) simplifies to

$$
C_{I}= \begin{cases}1 & \text { if } q<p \alpha, \\ 1 / 2\left(1+C_{I}\left[\mathcal{B}_{l}^{*}\right]\right) & \text { if } q=p \alpha, \\ 1 / 2 C_{I}\left[\mathcal{B}_{l}\right] & \text { if } q=w_{L}+p \alpha \\ 0 & \text { if } q>w_{L}+p \alpha\end{cases}
$$

$C_{I I}$ is obtained by replacing $p$ in (6) and (7) by $1-p$ and putting $C_{I I}=1-C_{I}$.

Proof The proof of Theorem 1 is available upon request. The main idea of the proof is as follows. Since we assume that the minor voters' choices are independent of one another, the aggregate voting weight of any coalition of minor voters is interpreted as a sum of independent random variables. This allows us to analyze the asymptotic properties of jury competence by means of a generalized central limit theorem, the Lindeberg-Feller theorem [see e.g., Theorem 4.7 in Petrov (1995) p. 123]. This method is valid as long as the weights of the minor voters are not too skewed, which is guaranteed by condition (2). The asymptotic behavior of jury competence begins to manifest itself at around 20 minor players. Estimates of convergence rates are available upon request. Figure 2 gives an illustration of Theorem 1. 


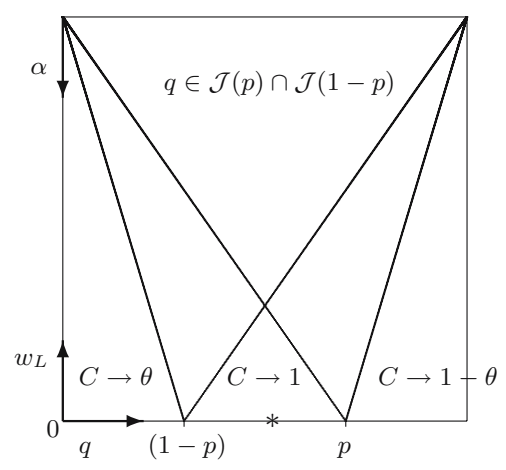

Fig. 2 Generalized Jury Theorem

In the triangle-shaped area around $q=1 / 2$ jury competence converges to infallibility, $C=1$, for lower values of $w_{L}$. Note that it contains the point marked with '*' on the horizontal $w_{L}=0$ (absence of major voters) and $q=1 / 2$. The simple majority rule, as considered by Condorcet, is a special case of this setting in which the block of votes, $\alpha=1$, is broken up and divided equally among an ever-increasing number of minor voters.

\section{References}

de Condorcet, N.C.: Essai sur l'application de l'analyse à la probabilité des décisions rendues à la pluralité des voix. Paris: De l'imprimerie royale (1785)

Dubey, P., Shapley L.S.: Mathematical properties of the Banzhaf power index. Math Oper Res 4(2), 99-131 (1979)

Fey, M.: A note on the Condorcet Jury Theorem with supermajority voting rules. Soc Choice Welfare 20(1), 27-32 (2003)

Petrov, V.V.: Limit theorems of probability theory. Oxford: Clarendon Press (1995)

Rae, D.W.: Decision rules and individual values in constitutional choice. Am Polit Sci Rev 63, 40-56 (1969) 